\title{
A SUBCLASS OF HARMONIC UNIVALENT FUNCTIONS WITH POSITIVE COEFFICIENTS
}

\author{
K. K. DIXIT AND SAURABH PORWAL
}

\begin{abstract}
Complex-valued harmonic functions that are univalent and sense-preserving in the open unit disc $U$ can be written in the form $f=h+\bar{g}$, where $h$ and $g$ are analytic in $U$. In this paper authors introduce the class, $R_{H}(\beta),(1<\beta \leq 2)$ consisting of harmonic univalent functions $f=h+\bar{g}$, where $h$ and $g$ are of the form $h(z)=z+\sum_{k=2}^{\infty}\left|a_{k}\right| z^{k}$ and $g(z)=\sum_{k=1}^{\infty}\left|b_{k}\right| z^{k}$ for which $\operatorname{Re}\left\{h^{\prime}(z)+g^{\prime}(z)\right\}<\beta$. We obtain distortion bounds extreme points and radii of convexity for functions belonging to this class and discuss a class preserving integral operator. We also show that class studied in this paper is closed under convolution and convex combinations.
\end{abstract}

\section{Introduction}

A continuous complex-valued function $f=u+i v$ is said to be harmonic in a simply connected domain $D$ if both $u$ and $v$ are real harmonic in $D$. In any simply connected domain we can write $f=h+\bar{g}$, where $h$ and $g$ are analytic in $D$. We call $h$ the analytic part and $g$ the co-analytic part of $f$. A necessary and sufficient condition for $f$ to be locally univalent and sense-preserving in $D$ is that $\left|h^{\prime}(z)\right|>\left|g^{\prime}(z)\right|, z \in D$. See Clunie and Sheil-Small [1].

Denote by $S_{H}$ the class of functions $f=h+\bar{g}$ that are harmonic univalent and sense-preserving in the unit disk $U=\{z:|z|<1\}$ for which $f(0)=f^{\prime}(0)-1=0$. Then for $f=h+\bar{g} \in S_{H}$ we may express the analytic functions $h$ and $g$ as

$$
h(z)=z+\sum_{k=2}^{\infty} a_{k} z^{k}, \quad g(z)=\sum_{k=1}^{\infty} b_{k} z^{k}, \quad\left|b_{1}\right|<1 .
$$

Note that $S_{H}$ reduces to the class of normalized analytic univalent functions if the co-analytic part of its member is zero.

A function $f$ of the form (1.1) is harmonic starlike for $|z|=r<1$, if

$$
\frac{\partial}{\partial \theta}\left(\arg f\left(r e^{i \theta}\right)\right)=\operatorname{Re}\left\{\frac{z h^{\prime}(z)-\overline{z g^{\prime}(z)}}{h(z)+g(z)}\right\}>0, \quad|z|=r<1 . \quad \text { See [2]. }
$$

Corresponding author: Saurabh Porwal.

Received November 18, 2008; revised December 4, 2009.

2000 Mathematics Subject Classification. 30C45, 30C50.

Key words and phrases. Harmonic, univalent, starlike, extreme points. 
Silverman [4] proved that the coefficient conditions $\sum_{k=2}^{\infty} k\left(\left|a_{k}\right|+\left|b_{k}\right|\right) \leq 1$ and $\sum_{k=2}^{\infty} k^{2}\left(\left|a_{k}\right|+\left|b_{k}\right|\right) \leq 1$ are sufficient conditions for functions $f=h+\bar{g}$ to be harmonic starlike convex functions, respectively.

Denote by $V_{H}$ the subclass of $S_{H}$ consisting of functions of the form $f=h+\bar{g}$, where

$$
h(z)=z+\sum_{k=2}^{\infty}\left|a_{k}\right| z^{k}, \quad g(z)=\sum_{k=1}^{\infty}\left|b_{k}\right| z^{k}, \quad\left|b_{1}\right|<1 .
$$

Recently Yalcin et al. [6] studied the class $H P(\alpha),(0 \leq \alpha<1)$ the subclass of $S_{H}$ satisfying the condition

$$
\operatorname{Re}\left\{h^{\prime}(z)+g^{\prime}(z)\right\}>\alpha .
$$

Further let $V_{H} P(\alpha)$ be the subclass of $V_{H}$ consisting of functions of the form (1.2) that satisfy condition (1.3).

Let $R_{H}(\beta),(1<\beta \leq 2)$, denote the subclass of $V_{H}$ satisfying the condition

$$
\operatorname{Re}\left\{h^{\prime}(z)+g^{\prime}(z)\right\}<\beta .
$$

We note that the class $R_{H}(\beta)$ reduces to class $R(\beta)$ if co-analytic part of $f$ is zero i.e. $g \equiv 0$ studied by Uralegaddi et al. [5]. Yalcin et al. [6] have studied the functions with negative coefficients that satisfy $\operatorname{Re}\left\{h^{\prime}(z)+g^{\prime}(z)\right\}>\alpha,(0 \leq \alpha<1)$ for $z \in U$. we need the following Lemma due to Theorem 2.1 of [6].

Lemma 1. Let $f=h+\bar{g} \in V_{H}$ be given by (1.2) and $\sum_{k=2}^{\infty} k\left|a_{k}\right|+\sum_{k=1}^{\infty} k\left|b_{k}\right| \leq 1-\alpha$, $(0 \leq \alpha<1)$ then $f \in V_{H} P(\alpha)$.

\section{Main results}

Theorem 2.1. A function $f$ of the form (1.2) is in $R_{H}(\beta)$ if and only if

$$
\sum_{k=2}^{\infty} k\left|a_{k}\right|+\sum_{k=1}^{\infty} k\left|b_{k}\right| \leq \beta-1
$$

Proof. Let $\sum_{k=2}^{\infty} k\left|a_{k}\right|+\sum_{k=1}^{\infty} k\left|b_{k}\right| \leq \beta-1$. It suffices to prove that

$$
\left|\frac{h^{\prime}(z)+g^{\prime}(z)-1}{h^{\prime}(z)+g^{\prime}(z)-(2 \beta-1)}\right|<1, \quad z \in U .
$$

We have $\left|\frac{h^{\prime}(z)+g^{\prime}(z)-1}{h^{\prime}(z)+g^{\prime}(z)-(2 \beta-1)}\right|$

$$
=\left|\frac{\sum_{k=2}^{\infty} k\left|a_{k}\right| z^{k-1}+\sum_{k=1}^{\infty} k\left|b_{k}\right| z^{k-1}}{\sum_{k=2}^{\infty} k\left|a_{k}\right| z^{k-1}+\sum_{k=1}^{\infty} k\left|b_{k}\right| z^{k-1}-2(\beta-1)}\right|
$$




$$
\begin{aligned}
& \leq\left|\frac{\sum_{k=2}^{\infty} k\left|a_{k}\right||z|^{k-1}+\sum_{k=1}^{\infty} k\left|b_{k}\right||z|^{k-1}}{2(\beta-1)-\sum_{k=2}^{\infty} k\left|a_{k}\right||z|^{k-1}-\sum_{k=1}^{\infty} k\left|b_{k}\right||z|^{k-1}}\right| \\
& \leq\left|\frac{\sum_{k=2}^{\infty} k\left|a_{k}\right|+\sum_{k=1}^{\infty} k\left|b_{k}\right|}{2(\beta-1)-\sum_{k=2}^{\infty} k\left|a_{k}\right|-\sum_{k=1}^{\infty} k\left|b_{k}\right|}\right|
\end{aligned}
$$

which is bounded above by 1 by hypothesis and the sufficient part is proved.

Conversely, suppose that

$$
\begin{aligned}
& \operatorname{Re}\left\{h^{\prime}(z)+g^{\prime}(z)\right\}<\beta, \quad \text { i.e. } \\
& \operatorname{Re}\left\{1+\sum_{k=2}^{\infty} k\left|a_{k}\right| z^{k-1}+\sum_{k=1}^{\infty} k\left|b_{k}\right| z^{k-1}\right\}<\beta, \quad z \in U .
\end{aligned}
$$

The above condition must hold for all values of $z,|z|=r<1$. Upon choosing the values of $z$ to be real and let $z \rightarrow 1^{-}$, we obtain

$$
\sum_{k=2}^{\infty} k\left|a_{k}\right|+\sum_{k=1}^{\infty} k\left|b_{k}\right| \leq \beta-1,
$$

which gives the necessary part. The proof of the theorem is complete.

Next we determine bounds for the class $R_{H}(\beta)$.

Theorem 2.2. If $f \in R_{H}(\beta)$, then

$$
|f(z)| \leq\left(1+\left|b_{1}\right|\right) r+\frac{1}{2}\left(\beta-1-\left|b_{1}\right|\right) r^{2}, \quad|z|=r<1
$$

and

$$
|f(z)| \geq\left(1-\left|b_{1}\right|\right) r-\frac{1}{2}\left(\beta-1-\left|b_{1}\right|\right) r^{2}, \quad|z|=r<1 .
$$

The bounds are sharp for the functions $f(z)=z+\left|b_{1}\right| \bar{z}+\frac{1}{2}\left(\beta-1-\left|b_{1}\right|\right) \bar{z}^{2}$ and $f(z)=$ $z+\left|b_{1}\right| \bar{z}+\frac{1}{2}\left(\beta-1-\left|b_{1}\right|\right) z^{2}$ for $\left|b_{1}\right| \leq \beta-1$.

Proof. Let $f \in R_{H}(\beta)$. Taking the absolute value of $f$, we have

$$
|f(z)| \leq\left(1-\left|b_{1}\right|\right) r+\sum_{k=2}^{\infty}\left(\left|a_{k}\right|+\left|b_{k}\right|\right) r^{k}
$$




$$
\begin{aligned}
& \leq\left(1+\left|b_{1}\right|\right) r+\sum_{k=2}^{\infty}\left(\left|a_{k}\right|+\left|b_{k}\right|\right) r^{2} \\
& \leq\left(1+\left|b_{1}\right|\right) r+\frac{1}{2} \sum_{k=2}^{\infty} k\left(\left|a_{k}\right|+\left|b_{k}\right|\right) r^{2} \\
& \leq\left(1+\left|b_{1}\right|\right) r+\frac{1}{2}\left(\beta-1-\left|b_{1}\right|\right) r^{2}
\end{aligned}
$$

and

$$
\begin{aligned}
|f(z)| & \geq\left(1+\left|b_{1}\right|\right) r-\sum_{k=2}^{\infty}\left(\left|a_{k}\right|+\left|b_{k}\right|\right) r^{k} \\
& \geq\left(1+\left|b_{1}\right|\right) r-\sum_{k=2}^{\infty}\left(\left|a_{k}\right|+\left|b_{k}\right|\right) r^{2} \\
& \geq\left(1+\left|b_{1}\right|\right) r-\frac{1}{2} \sum_{k=2}^{\infty} k\left(\left|a_{k}\right|+\left|b_{k}\right|\right) r^{2} \\
& \geq\left(1+\left|b_{1}\right|\right) r+\frac{1}{2}\left(\beta-1-\left|b_{1}\right|\right) r^{2} .
\end{aligned}
$$

The functions $z+\left|b_{1}\right| \bar{z}+\frac{1}{2}\left(\beta-1-\left|b_{1}\right|\right) \bar{z}^{2}$ and $z+\left|b_{1}\right| \bar{z}+\frac{1}{2}\left(\beta-1-\left|b_{1}\right|\right) z^{2}$ for $\left|b_{1}\right| \leq \beta-1$ show that the bounds given in Theorem 2.2 are sharp.

The following result follows from the left hand inequality in Theorem 2.2.

Corollary 2.1. If $f \in R_{H}(\beta)$, then

$$
\left\{\omega:|\omega|<\frac{1}{2}\left(3-\beta-\left|b_{1}\right|\right)\right\} \subset f(U) .
$$

Next we determine the extreme points of the closed convex hulls of $R_{H}(\beta)$, denoted by clco $R_{H}(\beta)$.

Theorem 2.3. $f \in \operatorname{clco} R_{H}(\beta)$, if and only if

$$
f(z)=\sum_{k=1}^{\infty}\left(\lambda_{k} h_{k}+\gamma_{k} g_{k}\right)
$$

where $h_{1}(z)=z, h_{k}(z)=z+\frac{\beta-1}{k} z^{k}(k=2,3,4, \ldots), g_{k}(z)=z+\frac{\beta-1}{k} \bar{z}^{k}(k=1,2,3, \ldots)$ and $\sum_{k=1}^{\infty}\left(\lambda_{k}+\gamma_{k}\right)=1, \lambda_{k} \geq 0$ and $\gamma_{k} \geq 0$. In particular the extreme points of $R_{H}(\beta)$ are $\left\{h_{k}\right\}$ and $\left\{g_{k}\right\}$.

Proof. For functions $f$ of the form (2.3) write

$$
f(z)=\sum_{k=1}^{\infty}\left(\lambda_{k} h_{k}+\gamma_{k} g_{k}\right)
$$




$$
=z+\sum_{k=2}^{\infty}\left(\frac{\beta-1}{k}\right) \lambda_{k} z^{k}+\sum_{k=1}^{\infty}\left(\frac{\beta-1}{k}\right) \gamma_{k} \bar{z}^{k} .
$$

Then

$$
\begin{aligned}
\sum_{k=2}^{\infty} & \frac{k}{\beta-1}\left(\frac{\beta-1}{k} \lambda_{k}\right)+\sum_{k=1}^{\infty} \frac{k}{\beta-1}\left(\frac{\beta-1}{k} \gamma_{k}\right) \\
& =\sum_{k=2}^{\infty} \lambda_{k}+\sum_{k=1}^{\infty} \gamma_{k} \\
& =1-\lambda_{1} \leq 1
\end{aligned}
$$

and so $f \in \operatorname{clcoR} R_{H}(\beta)$.

Conversely, suppose that $f \in \operatorname{clcoR}_{H}(\beta)$. Set $\lambda_{k}=\frac{k}{\beta-1}\left|a_{k}\right|,(k=2,3,4, \ldots)$ and $\gamma_{k}=$ $\frac{k}{\beta-1}\left|b_{k}\right|,(k=1,2,3, \ldots)$. Then note that by Theorem $2.1,0 \leq \lambda_{k} \leq 1,(k=2,3,4, \ldots)$ and $0 \leq \gamma_{k} \leq 1,(k=1,2,3, \ldots)$. We define $\lambda_{1}=1-\sum_{k=2}^{\infty} \lambda_{k}-\sum_{k=1}^{\infty} \gamma_{k}$ and note that by Theorem 2.1, $\lambda_{1} \geq 0$. Consequently, we obtain $f(z)=\sum_{k=1}^{\infty}\left(\lambda_{k} h_{k}+\gamma_{k} g_{k}\right)$ as required.

Theorem 2.4. If $f \in R_{H}(\beta)$ then $f \in V_{H} P(2-\beta)$.

Proof. The inclusion relation is a direct consequence of Lemma 1 and Theorem 2.1.

Next we give the interrelation between the class $R_{H}(\beta)$ and $S_{H}^{*}$, where $S_{H}^{*}$ is the class of harmonic starlike function in $U$.

Theorem 2.5. $R_{H}(\beta) \subseteq S_{H}^{*}$, where $1<\beta \leq 2$.

Proof. Let $f \in R_{H}(\beta)$. Then by Theorem 2.1

$$
\sum_{k=2}^{\infty} \frac{k}{\beta-1}\left|a_{k}\right|+\sum_{k=1}^{\infty} \frac{k}{\beta-1}\left|b_{k}\right| \leq 1 .
$$

Now

$$
\begin{aligned}
& \sum_{k=2}^{\infty} k\left|a_{k}\right|+\sum_{k=1}^{\infty} k\left|b_{k}\right| \\
& \quad \leq \sum_{k=2}^{\infty} \frac{k}{\beta-1}\left|a_{k}\right|+\sum_{k=1}^{\infty} \frac{k}{\beta-1}\left|b_{k}\right| \\
& \quad \leq 1 . \quad[\text { using }(2.4)]
\end{aligned}
$$

Thus $f \in S_{H}^{*}$.

This completes the proof of Theorem 2.5. 
Theorem 2.6. Each function in the class $R_{H}(\beta)$ maps a disks $U_{r}$ where $r<\inf _{k}$ $\left\{\frac{1}{k\left(\beta-1-\left|b_{1}\right|\right)}\right\}^{\frac{1}{k+1}}$ onto convex domains for $\beta>1+\left|b_{1}\right|$.

Proof. Let $f \in R_{H}(\beta)$ and let $r$, be fixed is that $0<r<1$, then $r^{-1} f(r z) \in R_{H}(\beta)$ and we have

$$
\begin{aligned}
\sum_{k=2}^{\infty} k^{2}\left(\left|a_{k}\right|+\left|b_{k}\right|\right) r^{k-1} & =\sum_{k=2}^{\infty} k\left(\left|a_{k}\right|+\left|b_{k}\right|\right)\left(k r^{k-1}\right) \\
& \leq \sum_{k=2}^{\infty} k\left(\left|a_{k}\right|+\left|b_{k}\right|\right) \\
& \leq \beta-1-\left|b_{1}\right| \leq 1
\end{aligned}
$$

provided

$$
\begin{aligned}
k r^{k-1} & \leq \frac{1}{\beta-1-\left|b_{1}\right|} \\
\text { or, } r & <\inf _{k}\left\{\frac{1}{k\left(\beta-1-\left|b_{1}\right|\right)}\right\}^{\frac{1}{k-1}} .
\end{aligned}
$$

The proof of Theorem 2.6 is complete.

For our next theorem, we need to define the convolution of two harmonic functions. For harmonic functions of the form

$$
f(z)=z+\sum_{k=2}^{\infty}\left|a_{k}\right| z^{k}+\sum_{k=1}^{\infty}\left|b_{k}\right| \bar{z}^{k}
$$

and

$$
F(z)=z+\sum_{k=2}^{\infty}\left|A_{k}\right| z^{k}+\sum_{k=1}^{\infty}\left|B_{k}\right| \bar{z}^{k}
$$

we define their convolution

$$
(f * F)(z)=f(z) * F(z)=z+\sum_{k=2}^{\infty}\left|a_{k} A_{k}\right| z^{k}+\sum_{k=1}^{\infty}\left|b_{k} B_{k}\right| \bar{z}^{k} .
$$

Using this definition, we show that the class $R_{H}(\beta)$ is closed under convolution.

Theorem 2.7. For $1<\beta \leq \alpha \leq 2$ let $f \in R_{H}(\alpha)$ and $F \in R_{H}(\beta)$. Then $f * F \in$ $R_{H}(\beta) \subseteq R_{H}(\alpha)$.

Proof. Let $f(z)=z+\sum_{k=2}^{\infty}\left|a_{k}\right| z^{k}+\sum_{k=1}^{\infty}\left|b_{k}\right| \bar{z}^{k}$ be in $R_{H}(\beta)$ and $F(z)=z+$ $\sum_{k=2}^{\infty}\left|A_{k}\right| z^{k}+\sum_{k=1}^{\infty}\left|B_{k}\right| \bar{z}^{k}$ be in $R_{H}(\alpha)$. Then the convolution $f * F$ is given by (2.5). We wish to show that the coefficients of $f * F$ satisfy the required condition given in 
Theorem 2.1. For $F(z) \in R_{H}(\alpha)$ we note that $\left|A_{k}\right| \leq 1$ and $\left|B_{k}\right| \leq 1$. Now, for the convolution function $f * F$, we have

$$
\begin{aligned}
& \sum_{k=2}^{\infty} \frac{k}{\beta-1}\left|a_{k} A_{k}\right|+\sum_{k=1}^{\infty} \frac{k}{\beta-1}\left|b_{k} B_{k}\right| \\
& \leq \sum_{k=2}^{\infty} \frac{k}{\beta-1}\left|a_{k}\right|+\sum_{k=1}^{\infty} \frac{k}{\beta-1}\left|b_{k}\right| \\
& \quad \leq 1 . \quad \text { (Science } f \in R_{H}(\beta) .
\end{aligned}
$$

Therefore $f * F \in R_{H}(\beta) \subseteq R_{H}(\alpha)$.

Next, we show that $R_{H}(\beta)$ is closed under convex combinations of its members.

Theorem 2.8. The class $R_{H}(\beta)$ is closed under convex combination.

Proof. For $i=1,2,3, \ldots$ let $f_{i}(z) \in R_{H}(\beta)$, where $f_{i}(z)$ is given by

$$
f_{i}(z)=z+\sum_{k=2}^{\infty}\left|a_{k_{i}}\right| z^{k}+\sum_{k=1}^{\infty}\left|b_{k_{i}}\right| \bar{z}^{k}
$$

Then by Theorem 2.1, we have

$$
\sum_{k=2}^{\infty} \frac{k}{\beta-1}\left|a_{k_{i}}\right|+\sum_{k=1}^{\infty} \frac{k}{\beta-1}\left|b_{k_{i}}\right| \leq 1
$$

For $\sum_{i=1}^{\infty} t_{i}=1,0 \leq t_{i} \leq 1$, the convex combination of $f_{i}$ may be written as

$$
\sum_{i=1}^{\infty} t_{i} f_{i}(z)=z+\sum_{k=2}^{\infty}\left(\sum_{i=1}^{\infty} t_{i}\left|a_{k_{i}}\right|\right) z^{k}+\sum_{k=1}^{\infty}\left(\sum_{i=1}^{\infty} t_{i}\left|b_{k_{i}}\right|\right) \bar{z}^{k}
$$

then by Theorem 2.1, we have

$$
\begin{aligned}
& \sum_{k=2}^{\infty} \frac{k}{\beta-1}\left(\sum_{i=1}^{\infty} t_{i}\left|a_{k_{i}}\right|\right)+\sum_{k=1}^{\infty} \frac{k}{\beta-1}\left(\sum_{i=1}^{\infty} t_{i}\left|b_{k_{i}}\right|\right) \\
& \quad=\sum_{i=1}^{\infty} t_{i}\left(\sum_{k=2}^{\infty} \frac{k}{\beta-1}\left|a_{k_{i}}\right|+\sum_{k=1}^{\infty} \frac{k}{\beta-1}\left|b_{k_{i}}\right|\right) \\
& \quad \leq \sum_{i=1}^{\infty} t_{i}=1
\end{aligned}
$$

Therefore

$$
\sum_{i=1}^{\infty} t_{i} f_{i}(z) \in R_{H}(\beta)
$$


The $\delta$-neighborhood of $f$ is the set

$$
\begin{aligned}
& N_{\delta}(f)=\left\{F: F(z)=z+\sum_{k=2}^{\infty}\left|A_{k}\right| z^{k}+\sum_{k=1}^{\infty}\left|B_{k}\right| \bar{z}^{k}\right. \\
& \left.\quad \text { and } \sum_{k=1}^{\infty} k\left(\left|a_{k}-A_{k}\right|+\left|b_{k}-B_{k}\right|\right) \leq \delta\right\} . \quad \text { See [3]. }
\end{aligned}
$$

Theorem 2.9. Let $f \in R_{H}(\beta)$ and $\delta \leq 2-\beta$. If $F \in N_{\delta}(f)$, then $F$ is harmonic starlike function.

Proof. Let $F(z)=z+\sum_{k=2}^{\infty}\left|A_{k}\right| z^{k}+\sum_{k=1}^{\infty}\left|B_{k}\right| \bar{z}^{k}$ belong to $N_{\delta}(f)$. We have

$$
\begin{aligned}
\sum_{k=2}^{\infty} k\left|A_{k}\right|+\sum_{k=1}^{\infty} k\left|B_{k}\right| & \leq \sum_{k=2}^{\infty} k\left(\left|a_{k}-A_{k}\right|+\left|b_{k}-B_{k}\right|\right)+\sum_{k=2}^{\infty} k\left(\left|a_{k}\right|+\left|b_{k}\right|\right)+\left|b_{1}-B_{1}\right|+\left|b_{1}\right| \\
& \leq \delta+\beta-1 \leq 1 .
\end{aligned}
$$

Hence, $F(z)$ is harmonic starlike function.

\section{A family of class preserving integral operator}

let $f(z)=h(z)+\overline{g(z)} \in S_{H}$ be given by (1.1) then $F(z)$ defined by relation

$$
F(z)=\frac{c+1}{z^{c}} \int_{0}^{c} t^{c-1} h(t) d t+\overline{\frac{c+1}{z^{c}} \int_{0}^{z} t^{c-1} g(t) d t}, \quad(c>-1) .
$$

Theorem 3.1. Let $f(z)=h(z)+\overline{g(z)} \in S_{H}$ be given by $(1.2)$ and $f(z) \in R_{H}(\beta)$ then $F(z)$ be defined by (3.1) also belong to $R_{H}(\beta)$.

Proof. Let $f(z)=z+\sum_{k=2}^{\infty}\left|a_{k}\right| z^{k}+\sum_{k=1}^{\infty}\left|b_{k}\right| z^{k}$ be in $R_{H}(\beta)$ then by Theorem 2.1, we have

$$
\sum_{k=2}^{\infty} \frac{k}{\beta-1}\left|a_{k}\right|+\sum_{k=1}^{\infty} \frac{k}{\beta-1}\left|b_{k}\right| \leq 1
$$

By definition of $F(z)$, we have

$$
F(z)=z+\sum_{k=2}^{\infty} \frac{c+1}{c+k}\left|a_{k}\right| z^{k}+\sum_{k=1}^{\infty} \frac{c+1}{c+k}\left|b_{k}\right| \bar{z}^{k} .
$$

Now

$$
\sum_{k=2}^{\infty} \frac{k}{\beta-1}\left(\frac{c+1}{c+k}\left|a_{k}\right|\right)+\sum_{k=1}^{\infty} \frac{k}{\beta-1}\left(\frac{c+1}{c+k}\left|b_{k}\right|\right)
$$




$$
\begin{aligned}
& \leq \sum_{\substack{k=2\\
}}^{\infty} \frac{k}{\beta-1}\left|a_{k}\right|+\sum_{k=1}^{\infty} \frac{k}{\beta-1}\left|b_{k}\right| \\
& \leq 1 .
\end{aligned}
$$

Thus $F(z) \in R_{H}(\beta)$.

\section{Acknowledgement}

The authors are thankful to referee for his valuable comments and suggestions. The present investigation was supposed by the University Grant Commission under grant No.11-12/2006(SA-I).

\section{References}

[1] J. Clunie and T. Sheil-Small, Harmonic univalent functions, Ann. Acad. Sci. Fen. Seriers A I Math., 9(1984), 3-25.

[2] J. M. Jahangiri, Harmonic functions starlike in the unit disk, J. Math. Anal. Appl., 235(1999), 470-477.

[3] St. Ruscheweyh, Neighborhoods of univalent functions, Proc. Amer. Math. Soc., 81(1981), $521-528$.

[4] H. Silverman, Harmonic univalent function with negative coefficients, J. Math. Anal. Appl. 220(1998), 283-289.

[5] B. A. Uralegaddi, M. D. Ganigi and S. M. Sarangi, Close-to-Convex functions with positive coefficients, Studia Univ. Babes-Bolyal, Mathematica XL, 4(1995), 25-31.

[6] Sibel Yalcin Karpuzoğullari, Metin Öztürk and Mümin Yamankaradeniz, A subclass of harmonic univalent functions with negative coefficients, Appl. Math. Comput., 142(2003), 469-476.

Department of Mathematics, Janta College, Bakewar, Etawah (U.P.) 206124, India.

Department of Mathematics, Janta College, Bakewar, Etawah (U.P.) 206124, India.

E-mail: saurabhjcb@rediffmail.com 